\title{
Assay concept for detecting anti-drug IgM in human serum samples by using a novel recombinant human IgM positive control
}

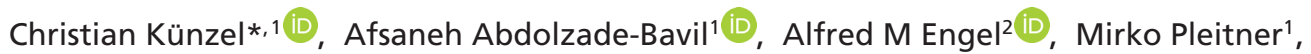 \\ Eginhard Schick ${ }^{3}$ \& Kay Stubenrauch ${ }^{1}$ (i) \\ ${ }^{1}$ Roche Pharma Research \& Early Development, Pharmaceutical Sciences, Bioanalytical R\&D, Roche Innovation Center Munich, Im \\ Nonnenwald 2, Penzberg, 82377, Germany \\ ${ }^{2}$ Centralized \& Point of Care Solutions, Roche Diagnostics GmbH, Nonnenwald 2, Penzberg, 82377, Germany \\ ${ }^{3}$ Roche Pharma Research \& Early Development, Pharmaceutical Sciences, Bioanalytical R\&D, Roche Innovation Center Basel, \\ Grenzacherstraße 124, Basel, CH-4070, Switzerland \\ *Author for correspondence: Tel.: +49 885660 7335; christian.kuenzel@roche.com
}

\begin{abstract}
Aim: Development and qualification of an easy-to-use ELISA for detection of IgM anti-drug antibodies (ADA) and its use in a clinical Phase I trial. Results \& methodology: During the assay development two positive control $(\mathrm{PC})$ approaches, the preparation of a chemically conjugated and a recombinant $\mathrm{PC}$, were pursued. With both PCs, the assay was developed and successfully qualified considering the regulatory guidelines. For a case study, the IgM ADA isotyping assay with the recombinant PC was selected. Different courses and intensities of immune response regarding IgM signals were demonstrated. Conclusion: The easy-to-use ELISA allowed IgM-ADA detection in clinical samples. Conjugated and recombinant IgM PCs were comparable regarding assay sensitivity, precision and suitability.
\end{abstract}

First draft submitted: 25 November 2020; Accepted for publication: 7 January 2021; Published online: 8 February 2021

Keywords: immunogenicity - ADA isotyping • IgM positive control • immune response characterization - biotherapeutics $\bullet$ targeted immunocytokine

Administration of a biopharmaceutical may lead to an unwanted immune response with loss of efficacy and safety concern including adverse events. Hence, immunogenicity assessment comprises an essential part of the development program for biopharmaceuticals, and supports the correct interpretation of pharmacokinetic, pharmacodynamic and safety data [1].

The initial encounter of the immune system with an antigen such as bio therapeutics triggers a primary immune response and a repeated contact with the same antigen induces the secondary immune response. During the primary immune response, naive B cells produce transiently IgM antibodies for about one to two weeks [2]. The immunoglobulin structure is pentameric, although monomer, tetramer and hexamer forms of IgM antibodies have been reported, too. IgM antibodies are less affine to the antigen with high functional avidity [3]. Due to their size $(900 \mathrm{kDa})$, structure and antigen binding properties, IgM antibodies are difficult to handle in laboratory work.

After repeated contact of the host with the same antigen, the antibody class switches to the IgG antibody produced by memory B cells [2]. The molecular weight of $\operatorname{IgG}$ antibodies is $150 \mathrm{kDa}$ with higher affinity and specificity to the antigen.

The immunogenicity assay package in clinical development includes at least screening, confirmation and titre assays. However, this approach does not provide any specific information on antibody characteristics. Therefore, additional anti-drug antibodies (ADA) characterization assays are beneficial to further investigate the ADA positive sample by neutralisation, isotyping or epitope/domain characterization assays. Gorovits recommends isotype characterization of ADA for particularly high immunogenicity risk biotherapeutics. He presents examples where understanding of isotyping and composition of ADA response (IgM, IgG1, IgG4 or IgE) can be highly important to predict future of disease progression or development of neutralizing ADAs [4]. Further ADA isotyping approach involving determination of immunoglobulin class, for example, IgG versus IgM of is described by Montjovent et al. [5]. 
(A)

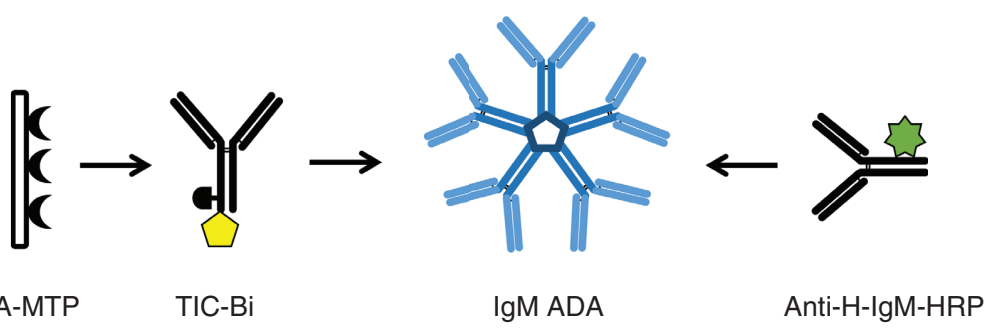

(B)

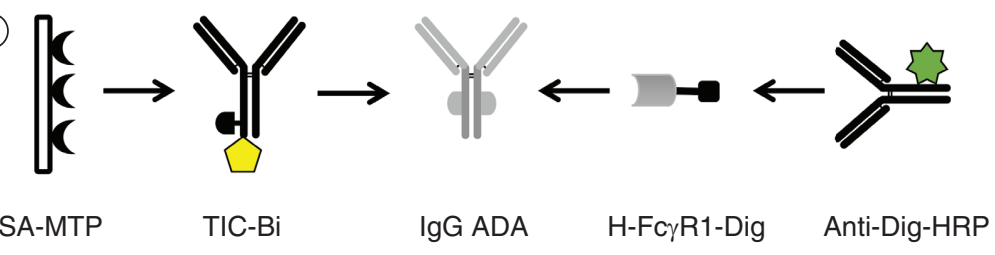

Figure 1. Schematic overview of the IgM and IgG isotyping enzyme-linked immunosorbent assays. (A) In the IgM ADA isotyping assay (AIA), the antigen binding sites of an IgM ADA binds to TIC-Bi coated on a SA-MTP. In the next step, the HRP coupled detection antibody binds to IgM ADA; (B) in the IgG AIA, the antigen binding site of an IgG ADA binds to TIC-Bi coated on a SA-MTP. Then, the human Dig-labelled FC RI binds to the IgG ADA followed by the final binding of the HRP coupled detection antibody.

ADA: Anti-drug antibody; Dig: Digoxigenin; HRP: Horseradish peroxidase; SA-MTP: Streptavidin coated microtiter plate; TIC-Bi: Biotinylated targeted immunocytokine.

To test human samples for IgG, IgM or other Ig isotypes, different platforms are provided by MSD, Bio-Rad and Genalyte. These platforms require a sample pre-treatment step to deplete the targeted ADAs of all isotypes. Leung et al. [6] described the Maverick detection system with MT-ADA assay kit from Genalyte for analyzing different isotypes. A biotin (Bi)-drug extraction combined with acid dissociation for elimination of matrix and drug interference was used by Niu et al. [7] for an AIA (ADA isotyping assay).

For development of an ELISA most reagents may be commercially sourced. Beside the capture and detection reagents, the positive control (PC) sample is one of the critical ADA assay tools and usually not commercially available. Different approaches may be used to generate a PC. In some instances, PC antibody may be purified from ADA positive subject samples [8]. Such human PCs are valuable assay tools but are not available at the early stage of trials, have limited volume, are less accepted due to ethical aspects and require patients' informed consent. Under these limitations, a long-term supply of subject-derived PCs seems impossible and does not fulfill the requirements of critical reagents for assays in life cycle management of biopharmaceutical programs.

Most frequently, PC antibodies are generated by immunizing animals, for example, rabbits. With this approach, polyclonal multi-epitope ADAs are generated. Polyclonal antibodies, generally in IgG form, are antigen affinity purified from animal's serum and are used as surrogate PC. Previously, a method described covalently cross-linking a monoclonal or polyclonal anti-idiotypic antibody to a human Ig with different isotypes [9]. Phage display offers another robust technology, which enables the identification of human anti-idiotype antibodies from naive scFv or Fab libraries in timeframes compatible with ADA assay development [10-12].

Often, monoclonal antibodies or panels thereof are used as PC reagent in ADA assays [8]. In this recombinant approach, first mice are immunized followed by subsequent cloning, production and purification of antibodies. Monoclonal ADAs are usually obtained in IgG form. Specific IgM antibodies may be generated by using different approaches such as recombinant monoclonal antibody engineering [13-15], whereby the $F(a b$ ') 2 part of the monoclonal anti-idiotypic is grafted into a human IgM antibody. Alternatively, chemically cross-linked conjugates can be generated by succinimide chemistry for the use as IgM PC antibodies $[9,16]$.

Most notably, the long-term availability of critical assay reagents is important for the life cycle management of the drug [17]. US FDA recommends sourcing a PC antibody for assessment of assay performance such as sensitivity, selectivity, specificity, drug tolerance and reproducibility (Figure 1) [8].

Here, we describe an easy to use homebrew IgM AIA without laborious sample pretreatments or acquisition of specific platform to characterize the immune response of ADA positive subjects. During the assay development, recombinant and conjugated PCs were evaluated as surrogate PC samples. In a case study, a human targeted 
immunocytokine (TIC) was administered to subjects. TIC is a cytokine coupled to Fc part of an IgG as described in [18]. ADA positive samples from this study were analyzed by the IgM AIA. Furthermore, an IgG AIA was used to complete the $\mathrm{ADA}$ isotyping results.

\section{Experimental}

\section{Chemicals, reagents \& equipment}

The following antibodies and assay reagents including PC samples were provided by Roche Diagnostics GmbH, Penzberg, Germany, otherwise it is mentioned. They were aliquoted and stored below $-60^{\circ} \mathrm{C}$ until use.

Peroxidase substrate 2,2'-azino-bis-3-ethylbenzthiazoline-6-sulphonic acid (\#11684302001), phosphatebuffered saline (PBS; \#11666789001) and Western Blocking Reagent (\#11921673001) were from Sigma Aldrich $\mathrm{GmbH}$ (Steinheim, Germany) and bovine serum albumin was in-house material. Low Cross Buffer ${ }^{\circledR}$ was commercially purchased from Candor Bioscience GmbH (Wangen, Germany) and used as assay buffer. PBS with $0.5 \%$ bovine serum albumin (PBS+) and Western Blocking Reagent TBS-Tween were used in assay development. Western Blocking Reagent TBS-Tween was prepared according to manufacturers' instructions. Tris base (Tris[hydroxymethyl]-aminomethane) was commercially purchased from Merck KGaA (Darmstadt, Germany; \#1083821000). For the chemical conjugated PC, a monoclonal mouse anti-TIC IgG and a nonspecific binding polyclonal human IgM from an internal source were used.

Human pool serum and individual human serum samples collected from healthy individuals were obtained from Sera Laboratory International Ltd. (Haywords Heath, West Sussex, UK).

Streptavidin (SA) coated polystyrene 96-well ELISA microtiter plates (SA-MTP) were provided by Roche Diagnostics GmbH (Mannheim, Germany; \#11643673001). For pre-incubation steps a polypropylene MTP was used from Thermo Fisher Scientific Inc. (MA, USA; \#267245).

The washing buffer contained 0.05\% Tween-20 (Calbiochem, Darmstadt, Germany) in PBS.

\section{PC reagents}

The following compounds served as PC: For the IgM AIA, a conjugate of a mouse monoclonal anti-TIC with a polyclonal human IgM antibody as well as a recombinant IgM (preparation see below). The production of the chemically conjugated PC (hereinafter referred to as conjugated PC) has been already described [13].

Sequence analysis of variable heavy and light chains of monoclonal anti-TIC mouse IgG was performed by RACE PCR after mRNA isolation by using 5'/3'RACE kit 2nd Gen (Roche Diagnostics GmbH) and PCR primers as described in literature [11]. The PCR amplicons were directly sequenced using the same primers after purification. The mouse variable coding regions were fused to human immunoglobulin gamma-1 heavy chain (sw_hum:IGG1_HUMAN), immunoglobulin mu constant chain C region (sw_hum:IGHM_human) and immunoglobulin kappa constant (sw_hum:IGKC_HUMAN) coding regions, to yield chimeric mouse/human IgG1 and IgM expression constructs. All immunoglobulin expression constructs were subcloned via BsmI in-frame to the same variable heavy chain mouse leader sequence featuring an intron as already described [15]. Expression was under the control of a human cytomegalovirus immediate-early enhancer/promoter region followed by a bovine growth hormone polyadenylation signal. For expression of the IgM, a human J chain was co-transfected. Therefore, the human immunoglobulin J chain (sw_hum:IGJ_HUMAN) coding region was subcloned into pEF6/V5 downstream of an EF-1a promoter.

For transient gene expression in human embryonic kidney (HEK) 293 cells, the FreeStyle ${ }^{\mathrm{TM}}$ 293-F expression system (Thermo Fischer Scientific, MA, USA) was used as described previously [19].

\section{Clinical samples}

Serum samples for ADA analysis were collected from subjects enrolled in a Phase I clinical trial for the treatment of patients with advanced and/or metastatic solid tumours. In this trial, subjects were submitted to a multiple intravenous dosage. Patient's informed consent was available for these investigations.

ADA samples were taken at different timepoints as shown in Figure 3. In case no ADA samples were available, corresponding PK (pharmacokinetics) samples were used instead. In total, 57 samples from 18 subjects were confirmed positive for the presence of ADA. All available samples from these subjects were further characterized by IgM and IgG AIA. 


\section{General method descriptions}

All antibody reagent solutions were prepared in assay buffer. Reagents and samples were incubated at room temperature on MTP with shaking at 500 r.p.m. MTPs were washed three-times by dispensing and aspirating $300 \mu \mathrm{l}$ of washing buffer in each MTP well.

After adding the substrate 2,2'-azino-bis-3-ethylbenzthiazoline-6-sulphonic acid, the horseradish peroxidase (HRP) catalyses a color reaction with spectrophotometric readout at $405 \mathrm{~nm}$ wavelength (reference wavelength: $490 \mathrm{~nm}$ ). Assay read out was reported as absorbance unit (AU). Each sample was analyzed as duplicate. The absorbance values were averaged and accepted if the precision of the mean value was $\leq 20 \%$ coefficient of variation $(\% \mathrm{CV})$.

While for screening and confirmatory assay fully validated assays were used, the $\operatorname{IgG}$ and $\operatorname{IgM}$ isotyping was performed under exploratory conditions using qualified assays.

A fit-for-purpose assay qualification was conducted to investigate the assay parameters including intra- and interassay precision, cut point (CP), drug tolerance, specificity and selectivity. All parameters fulfilled the predefined acceptance criteria. Furthermore, the sensitivity, defined as the concentration at which the antibody preparation produces an assay readout equal to the $\mathrm{CP}$ determined for that particular assay, was investigated. All assay parameters were evaluated according to the guidelines [1].

\section{ADA screening \& confirmation assay}

The ADA screening and confirmatory assays were evaluated as described by Shankar et al. [20].

\section{$\lg \mathrm{M}$ AIA}

The IgM AIA was a serial sandwich ELISA with an minimum required dilution (MRD) of 1:100. The Bi labeled TICs with an assay concentration of $1 \mu \mathrm{g} / \mathrm{ml}$ was used as capture reagent. As detection reagent in the IgM AIA, a polyclonal antibody from goat directed against human IgM antibodies coupled with HRP, \#R1340HRP from OriGene Technologies Inc (MA, USA) was used. The stock solution was diluted 1:2,500.

At the first step, the capture reagent TIC-Bi $(0.5 \mu \mathrm{g} / \mathrm{ml})$ was incubated for $1 \mathrm{~h}$ on a SA-MTP followed by the washing step. Then, $2.5 \mu \mathrm{l}$ of PC, NC or test samples was diluted by adding to $247.5 \mu \mathrm{l}$ of assay buffer. This mixture was added to the TIC-Bi coated SA-MTP and incubated for $1 \mathrm{~h}$. After incubation, the washing step was repeated. The detection reagent was added and incubated for $1 \mathrm{~h}$ followed by another washing step. Finally, the substrate solution was pipetted to the wells and colorimetric signal was detected. Samples containing IgM antibodies against TIC generated an assay signal with proportional intensity to the present amount of IgM ADA.

\section{$\operatorname{IgG}$ AIA}

The IgG AIA was a serial sandwich ELISA with an MRD of 1:50. At the first step, the capture reagent TIC-Bi $(0.5 \mu \mathrm{g} / \mathrm{ml})$ was incubated for $1 \mathrm{~h}$ on a SA-MTP followed by the washing step. Then, $5 \mu \mathrm{l}$ of PC, NC or test samples was diluted by adding to $245 \mu \mathrm{l}$ of assay buffer. This mixture was added to the TIC-Bi coated SA-MTP and incubated for $1 \mathrm{~h}$. After incubation, the washing step was repeated. A human Fc R1 (CD64), \#MAB1257 from R\&D Systems Inc. (MN, USA) was digoxygenin (Dig) labeled and used as described by Wessels et al. [21] as first detection reagent. This Fc R1, which binds IgG1, IgG3 and IgG4, was chosen to detect as many IgG subtypes as possible. The first detection reagent was added and incubated for $1 \mathrm{~h}$ followed by another washing step. The second detection reagent was anti-Dig-HRP.

\section{Results \& discussion}

\section{IgM AlA assay development \& optimization}

The optimal assay conditions and key assay parameters were established prior to assay qualification.

The TIC-Bi coated to a SA-MTP captures all ADA and the detection reagent subsequently distinguishes between the isotypes. For the developed IgM AIA, a polyclonal anti-human IgM coupled with HRP is directly applied as detection reagent. In the parallel applied IgG AIA, the first detection reagent Dig-labelled Fc RI (CD64) binds to the IgG ADA followed by binding of second detection reagent, the HRP coupled polyclonal anti-Dig antibody (Figure 1).

Due to the 'stickiness' and the unspecific binding of IgM antibodies causing high signal to noise ratio, it is challenging to develop a sensitive and specific IgM detection assay. Therefore, the evaluation of appropriate MTP, assay buffer and capture/detection reagents and their concentrations is essential for assay development. 
Table 1. Selection of assay buffer based on investigation of matrix effects.

\begin{tabular}{|c|c|c|c|c|c|c|c|}
\hline \multirow[t]{3}{*}{ Sample } & \multirow{3}{*}{$\begin{array}{l}\text { PC assay conc. } \\
(\mathrm{ng} / \mathrm{mL})\end{array}$} & \multicolumn{6}{|c|}{ Signal (AU) } \\
\hline & & \multicolumn{2}{|c|}{ PBS+ matrix } & \multicolumn{2}{|c|}{ WBRT matrix } & \multicolumn{2}{|c|}{ LCB matrix } \\
\hline & & $0 \%$ & $1 \%$ & $0 \%$ & $1 \%$ & $0 \%$ & $1 \%$ \\
\hline PC3 & 20 & 2.051 & 2.485 & 2.366 & 2.567 & 1.913 & 2.051 \\
\hline PC2 & 10 & 1.194 & 1.706 & 1.396 & 1.772 & 1.055 & 1.277 \\
\hline PC1 & 5 & 0.636 & 1.136 & 0.766 & 1.159 & 0.562 & 0.814 \\
\hline NC & 0 & 0.060 & 0.480 & 0.052 & 0.467 & 0.042 & 0.255 \\
\hline
\end{tabular}

Assay buffers were tested in the absence and presence of different concentrations of recombinant PC and with or without matrix.

AU: Absorbance units; LCB: Low cross buffer; NC: Negative control; PBS: Phosphate-buffered saline; PBS+: PBS containing 0.5\% bovine serum albumin; PC: Positive control; WBRT: Western blocking reagent TBS-Tween.

Figure 2. Comparison of assay signals obtained for phosphate-buffered saline+ and low cross buffer in the IgM anti-drug antibody isotyping assay. The signals of serum samples obtained from 40 healthy donors are compared PBS+ and LCB. LCB shows clearly lower assay signal and variability.

LCB: Low cross buffer; NC: Negative control; PBS: Phosphate-buffered saline.

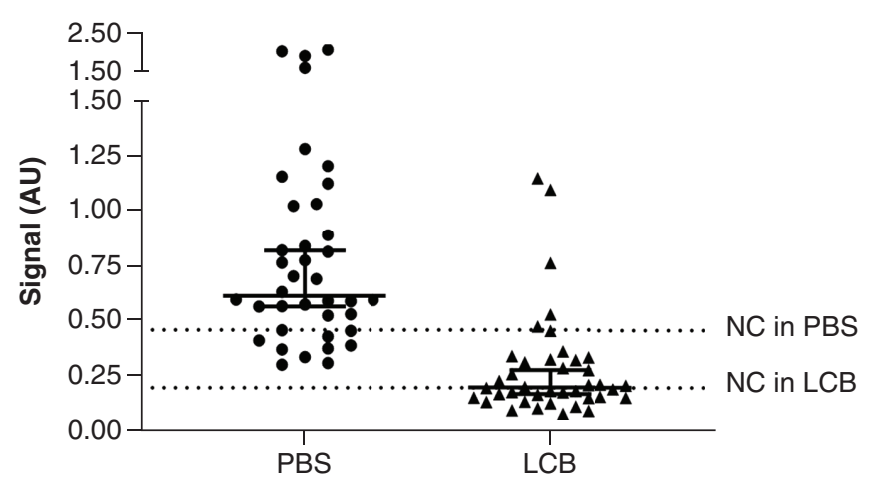

\section{Assay buffer \& MTP selection}

Three assay buffers were tested with different concentrations of the recombinant PC and with or without matrix (Table 1). In absence of matrix, the assay buffers PBS+, Western blocking reagent TBS-Tween and low cross buffer (LCB) showed comparable assay signals. In presence of matrix, the negative control signals increased approximately tenfold. The LCB showed the best signal-to-noise ratio of 3.2, 5.0 and 8.0 for PC1, PC2 and PC3 in presence of $1 \%$ matrix. The evaluation of 40 naive human individual sera (Figure 2) show a high assay signal variation with $\mathrm{PBS}+$ resulting in a high assay CP at upper limit of the assay. Whereas the use of LCB as assay buffer resulted in a low assay signal distribution with an applicable CP (Figure 2).

For this assay, the streptavidin coated MTPs were used to reduce nonspecific binding and minimize assay background signal. Biotin labeled TIC was immobilized on the streptavidin coated MTP building a specific capture basis for the immunoassay.

\section{Capture \& detection reagent concentrations}

TIC-Bi was applied as capture reagent to enable specific binding of all ADA isotypes. Different anti-IgM antibodies from several vendors were tested in the IgM AIA assay set up. The anti-IgM antibody with the lowest signal-to-noise ratio was selected (data not shown). Different ratios of detection and capture reagent at various concentrations were tested using the recombinant $\mathrm{PC}$ as test sample (Table 2). The best signal-to-noise $(\mathrm{S} / \mathrm{N})$ ratio was determined with $0.5 \mathrm{mg} / \mathrm{ml}$ of TIC-Bi and 1:2,500 dilution of anti-IgM HRP.

\section{Minimum required dilution}

The MRD is the minimum dilution necessary for the most sensitive detection of ADA in biological matrix with least interference. For this purpose, the $\mathrm{S} / \mathrm{N}$ ratio of spiked $\mathrm{PC}$ to $\mathrm{NC}$ is calculated to evaluate the best $\mathrm{MRD}$. In this experiment, the PC was prepared in assay buffer containing $0,1,2,5$ and $10 \%$ human serum. As shown in Table 3, the $1 \%$ assay matrix revealed an $\mathrm{S} / \mathrm{N}$ of 4.5 . Therefore, the MRD applied in the IgM AIA was set to 100.

\section{Assay qualification \& PC evaluation}

Before initiation of clinical samples analysis, the IgM and IgG AIA were qualified regarding key assay parameters such as sensitivity, intra- and inter-assay precision, specificity, and selectivity. Selected qualification data of the IgM AIA is summarized in Tables $4 \& 5$. 
Table 2. Testing of different ratios for detection and capture reagent for IgM anti-drug antibody isotyping assay.

\begin{tabular}{|c|c|c|c|c|c|c|c|c|c|c|c|c|c|}
\hline \multirow[t]{3}{*}{$\begin{array}{l}\text { Detection reagent } \\
\text { anti-IgM-HRP }\end{array}$} & \multirow[t]{3}{*}{$\begin{array}{l}\text { Recombinant PC } \\
(\mathrm{ng} / \mathrm{ml})\end{array}$} & \multicolumn{12}{|c|}{$\begin{array}{l}\text { Capture reagent } \\
\text { TIC-Bi }(\mu \mathrm{g} / \mathrm{ml})\end{array}$} \\
\hline & & \multicolumn{2}{|r|}{4.0} & \multicolumn{2}{|r|}{1.0} & \multicolumn{2}{|r|}{0.5} & \multicolumn{2}{|r|}{0.13} & \multicolumn{2}{|r|}{0.03} & \multicolumn{2}{|r|}{0.00} \\
\hline & & $S / N$ & Signal (AU) & $S / N$ & Signal (AU) & $S / N$ & Signal (AU) & $S / N$ & Signal (AU) & $\mathrm{S} / \mathrm{N}$ & Signal (AU) & $S / N$ & Signal (AU) \\
\hline \multirow[t]{4}{*}{$1: 20,000$} & 2000 & 3.4 & 0.710 & 6.6 & 0.671 & 7.7 & 0.600 & 5.9 & 0.394 & 2.9 & 0.194 & 1.0 & 0.066 \\
\hline & 1000 & 2.4 & 0.502 & 4.3 & 0.438 & 4.8 & 0.376 & 3.7 & 0.249 & 2.0 & 0.133 & 1.1 & 0.067 \\
\hline & 500 & 1.9 & 0.389 & 2.8 & 0.281 & 3.0 & 0.232 & 2.3 & 0.153 & 1.4 & 0.096 & 1.0 & 0.065 \\
\hline & 0 & 1.0 & 0.206 & 1.0 & 0.102 & 1.0 & 0.078 & 1.0 & 0.067 & 1.0 & 0.067 & 1.0 & 0.063 \\
\hline \multirow[t]{4}{*}{$1: 10,000$} & 2000 & 4.0 & 1.454 & 7.5 & 1.251 & 9.0 & 1.117 & 7.5 & 0.740 & 3.5 & 0.335 & 1.0 & 0.098 \\
\hline & 1000 & 2.8 & 0.996 & 4.9 & 0.818 & 5.8 & 0.722 & 4.6 & 0.452 & 2.3 & 0.220 & 1.0 & 0.098 \\
\hline & 500 & 2.0 & 0.704 & 2.9 & 0.489 & 3.5 & 0.435 & 2.8 & 0.274 & 1.6 & 0.153 & 0.9 & 0.094 \\
\hline & 0 & 1.0 & 0.360 & 1.0 & 0.167 & 1.0 & 0.124 & 1.0 & 0.099 & 1.0 & 0.097 & 1.0 & 0.100 \\
\hline \multirow[t]{4}{*}{ 1:5000 } & 2000 & 4.4 & 2.389 & 8.9 & 2.116 & 10.7 & 1.895 & 7.4 & 1.202 & 3.2 & 0.527 & 1.0 & 0.149 \\
\hline & 1000 & 3.0 & 1.601 & 5.7 & 1.359 & 6.5 & 1.159 & 4.2 & 0.689 & 2.0 & 0.329 & 1.0 & 0.147 \\
\hline & 500 & 2.1 & 1.158 & 3.5 & 0.827 & 3.8 & 0.676 & 2.4 & 0.396 & 1.3 & 0.219 & 1.0 & 0.157 \\
\hline & 0 & 1.0 & 0.541 & 1.0 & 0.237 & 1.0 & 0.177 & 1.0 & 0.163 & 1.0 & 0.163 & 1.0 & 0.153 \\
\hline \multirow[t]{4}{*}{ 1:2500 } & 2000 & 3.8 & 3.145 & 8.4 & 3.205 & 13.0 & 3.001 & 9.8 & 2.215 & 3.9 & 0.940 & 0.8 & 0.220 \\
\hline & 1000 & 3.4 & 2.746 & 6.1 & 2.338 & 7.8 & 1.801 & 4.3 & 0.986 & 2.3 & 0.560 & 0.9 & 0.259 \\
\hline & 500 & 2.5 & 2.035 & 3.8 & 1.434 & 5.0 & 1.157 & 3.2 & 0.728 & 1.5 & 0.365 & 0.9 & 0.257 \\
\hline & 0 & 1.0 & 0.818 & 1.0 & 0.382 & 1.0 & 0.231 & 1.0 & 0.227 & 1.0 & 0.243 & 1.0 & 0.285 \\
\hline
\end{tabular}

Different ratios of detection and capture reagent were tested with different concentrations of $P C$ as test sample.

AU: Absorbance unit; pAb: Polyclonal antibody; PC: Positive control; S/N: Signal-to-noise ratio; TIC-Bi: Biotinylated targeted immunocytokine.

\section{Table 3. Evaluation of the minimum required dilution for lgM anti-drug antibody isotyping assay. \\ Variables \\ Minimum required dilution}

\begin{tabular}{|c|c|c|c|c|c|}
\hline & \\
\hline & 0 & 100 & 50 & 20 & 10 \\
\hline Matrix & $0 \%$ & $1 \%$ & $2 \%$ & $5 \%$ & $10 \%$ \\
\hline Assay buffer & $100 \%$ & $99 \%$ & $98 \%$ & $95 \%$ & $90 \%$ \\
\hline PC (signal), AU & 2.061 & 1.998 & 2.054 & 2.050 & 2.086 \\
\hline $\mathrm{NC}$ (noise), AU & 0.083 & 0.446 & 0.753 & 1.216 & 1.611 \\
\hline $\mathrm{S} / \mathrm{N}$ & 24.8 & 4.5 & 2.7 & 1.7 & 1.3 \\
\hline
\end{tabular}

Different dilutions were tested with $9.2 \mathrm{ng} / \mathrm{ml}$ recombinant $\mathrm{PC}$ in comparison to samples without recombinant $\mathrm{PC}$. Based on these results, the $\mathrm{S} / \mathrm{N}$ was calculated

AU: Absorbance units; NC: Negative control; PC: Positive control; S/N: Signal-to-noise ratio.

\section{Table 4. Qualification data of the IgM assay using two different positive controls.}

Parameters

Sensitivity at CP in serum $(\mathrm{ng} / \mathrm{ml})$

Specificity

Normalization factor

Intra-assay precision (\%CV)

Inter-assay precision (\%CV)

Selectivity (\%RE)

The conjugated and the recombinant PCs, two differently prepared positive controls were evaluated in parallel. The intra- and inter-assay precision is expressed as coefficient of variation. For the IgM conjugated PC, the control levels were prepared at 4000, 7300 and 15,000 ng/ml and for the recombinant PC at 400,730 and $1500 \mathrm{ng} / \mathrm{ml}$. For the IgG conjugated PC, the control levels were prepared at 469, 625 and $1870 \mathrm{ng} / \mathrm{ml}$ and for the recombinant PC at 234, 420 and $1870 \mathrm{ng} / \mathrm{ml}$.

AIA: ADA isotyping assay, PC: Positive control; CP: Cut point; \%CV: Coefficient of variation.
IgM AIA

Conjugated PC

$3540(n=1)$

No cross reactivity to $\lg G$ or $\lg \mathrm{E}$

2.18

$1.4-3.5(n=3)$

$5.6-6.6(n=3)$

$3.6-6.6(n=3)$

\section{Recombinant PC}

$354(n=1)$

No cross reactivity to $\lg G$ or $\lg E$

2.18

1.5-4.5 $(n=5)$

$5.0-10.5(n=6)$

5.8-19.5 $(n=11)$

The assay sensitivity is defined as $\mathrm{PC}$ concentration at $\mathrm{CP}$. Compared with the conjugated PC, the sensitivity of the IgM AIA determined with the recombinant PC was tenfold higher. The conjugated PC is prepared by crosslinking the IgM molecule $(900 \mathrm{kDa})$ with an $\mathrm{IgG}$ molecule $(150 \mathrm{kDa})$ yielding in a multimer of $1050 \mathrm{kDa}$ or 
Table 5. Evaluation of the drug tolerance using the recombinant positive controls.

\begin{tabular}{|c|c|c|c|c|c|c|c|c|}
\hline \multirow{2}{*}{$\begin{array}{l}\text { IgM ADA } \\
(\mu \mathrm{g} / \mathrm{ml})\end{array}$} & \multicolumn{8}{|c|}{ TIC (ng/ml) } \\
\hline & 200000 & 100000 & 50000 & 25000 & 12500 & 6300 & 3100 & 0 \\
\hline 5.00 & 0.593 & 0.834 & 1.188 & 1.609 & 2.066 & 2.564 & 2.696 & 2.809 \\
\hline 2.50 & 0.436 & 0.561 & 0.735 & 0.931 & 1.186 & 1.626 & 1.953 & 2.215 \\
\hline 1.25 & 0.361 & 0.412 & 0.511 & 0.607 & 0.721 & 0.922 & 1.177 & 1.528 \\
\hline 0 & 0.281 & 0.279 & 0.276 & 0.273 & 0.278 & 0.273 & 0.272 & 0.273 \\
\hline
\end{tabular}

For both assays, the recombinant PC was applied. The cut point for the IgM ADA isotyping assay is 0.6314 and for the IgG ADA isotyping assay 0.2761 . All test samples with signals above the cut point are ADA positive and highlighted in green

ADA: Anti-drug antibody; PC: Positive control; TIC: Targeted immunocytokine.

higher. The size and steric hindrance of conjugated PC may be a reason for lower sensitivity of the assay. Based on the ELISA data and considering the molecular mass of both PCs, comparable affinities to TIC are expected.

Specificity was demonstrated for IgM AIA with both PCs tested and no cross reactivity was found to IgG (data in Supplementary Figure 3).

The intra- and inter-assay precision were comparable for both types of PC and within acceptable criteria of $\pm 20 \%$. To determine the selectivity, 11 individual healthy donor serum samples were spiked at three PC levels and were analyzed for IgM. The recovery of PC was investigated by calibration curve using the respective PC as reference material. Concentration of samples at all three PC levels were comparable for the conjugated and the recombinant PC in IgM AIA and within acceptable criteria of $\pm 20 \%$ of nominal.

The assay specific CP was evaluated according to Shankar et al., using the $99 \%$ percentile (1\% false positive rate) applied to 34 drug naive donor samples [18]. The normalization factor (NF) is the CP divided by the average signal of three NCs. The plate specific CP was calculated by multiplying the NF with the blank signal of the NC. Samples with signals below the plate-specific CP were rated as negative. Samples with signals equal or above the plate-specific $\mathrm{CP}$ were rated as positive. It was conducted by one analyst in three runs using 34 healthy individual samples. The results are shown in Figure 2. A nonparametrical distribution was found for both AIAs and no outliers were excluded. For the IgM assay, a higher assay background signal was detected for the CP results. The $\mathrm{CP}$ evaluation resulted in $0.745 \mathrm{AU}(\mathrm{NC}=0.342 \mathrm{AU})$ for IgM AIA. By using a NF a plate specific cut point was calculated for each plate by multiplying the NF to the NC.

\section{Drug tolerance}

Another critical step during assay development was the investigation of assay drug tolerance to achieve the best possible assay sensitivity. For evaluation of drug tolerance recombinant PCs were used. Different concentrations of TIC were spiked to a dilution series of IgM ADA PCs. The results of the drug tolerance assessment are shown in Table 5. As result, $1250 \mathrm{ng} / \mathrm{ml}$ IgM ADA could be detected at $12.5 \mu \mathrm{g} / \mathrm{ml} \mathrm{TIC} \mathrm{concentration.} \mathrm{However,} \mathrm{in} \mathrm{the}$ case study the detected TIC levels were below $12.5 \mu \mathrm{g} / \mathrm{ml}$ and the IgM AIA signals were not affected by drug as shown in Figure 3.

\section{Assay selection for case study}

For the case study, we selected as analyzing format ELISAs for the IgM and IgG AIA and the recombinant approach as PC. The advantage of an ELISA is the simple assay principle with easy accessible reagents that can be carried out in any laboratory without the need for special equipment. While technical data of both PC approaches was comparable as described above, the recombinant $\mathrm{PC}$ was selected based on the well-defined manufacturing process and negligible batch-to-batch variability. This assay system will ensure a long term supply of reagents and simplify transfer of assays to external laboratories.

\section{Case study}

The developed IgM AIA was used for studying the immune response of individuals during a Phase I study receiving repeated dose of TIC. In this study, 18 out of 110 treated subjects developed an immune response against the administered drug. For the majority of the subjects the ADA onset was already detected during the first treatment cycle, 4 days after first drug administration. The early immune response was an indication for a treatment-induced IgM response. To prove this hypothesis, all available samples of ADA confirmed subjects were further analyzed by 
(A)

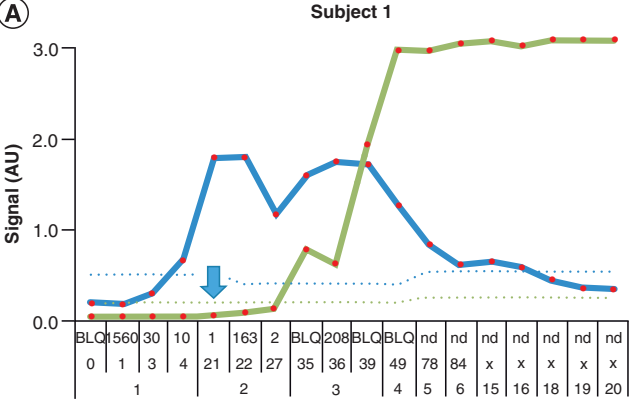

(c)

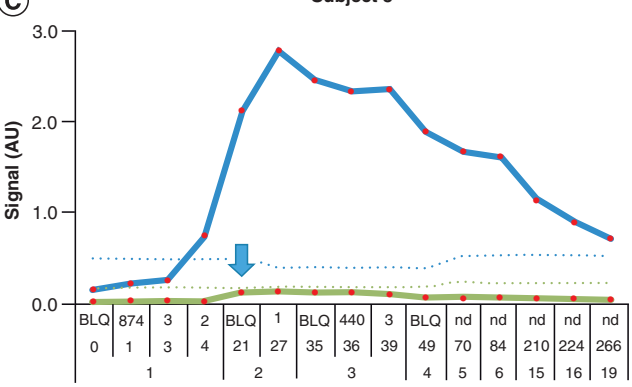

(E)

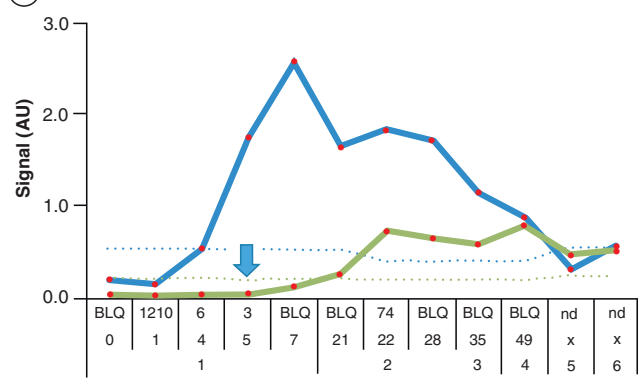

(B)

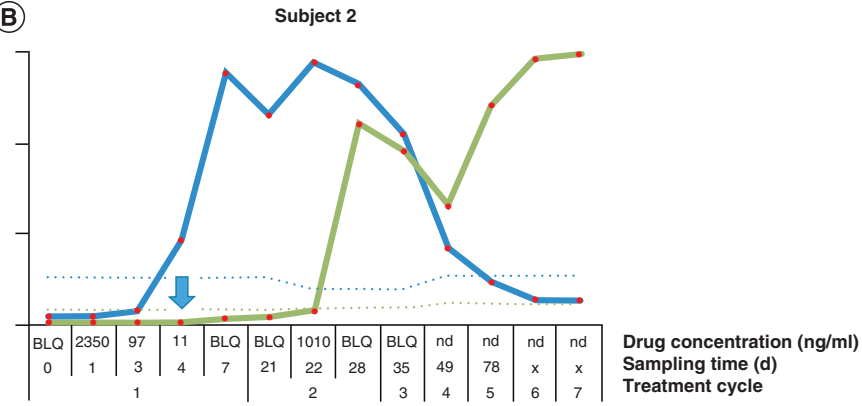

(D)

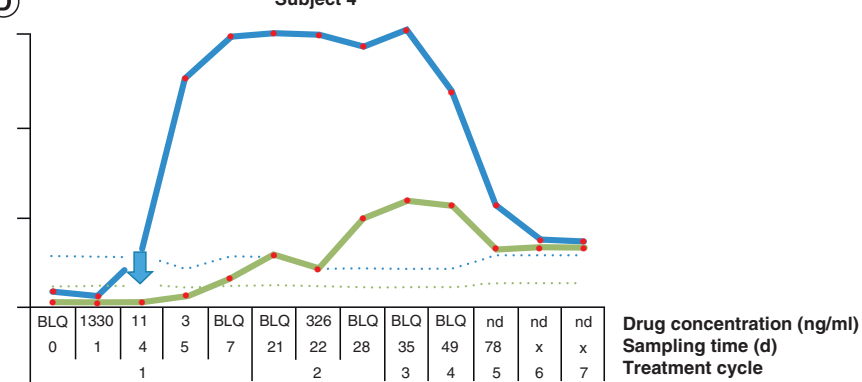

(

Subject 6

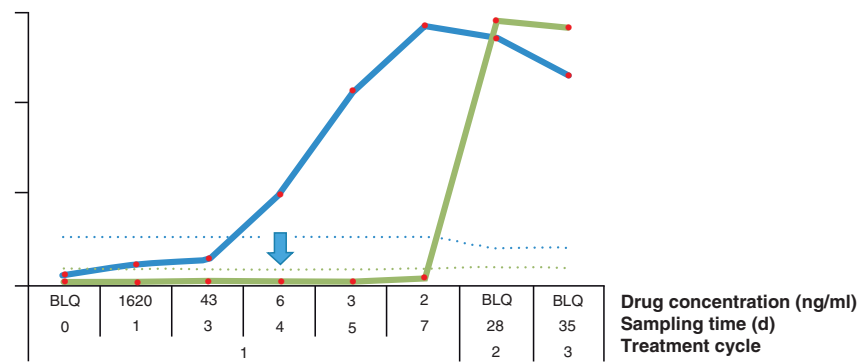

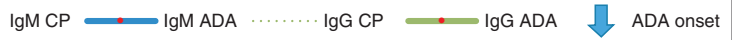

Figure 3. Strong immune response detected by $\lg M$ and $\lg G$ anti-drug antibody isotyping assay for six subjects in the case study. (A) For subject 1, from study day 4 onwards a moderate ADA IgM response is detected. An immunoglobulin class switch is observed on day 39 with very high persistent ADA IgG signal. (B) For subject 2 the IgM ADA signal was above the cut point on day 4 after the first TIC administration. The signal reached a plateau on day 7 with decreasing signal from day 28 onwards. Altering IgG ADA signal was detected in cycle 2 after second TIC dose from day 28 onwards. (C) For subject 3 only IgM ADA response was detected 4 days after the first TIC administration reaching a maximum in cycle 2 . The IgM ADA signal was above the assay cut point until last time point tested on study day 266 in cycle 19. For all time points the IgG ADA isotyping assay signal was below the assay cut point. (D) For subject 4 a very high IgM ADA signal was detected after 4 days the signal intensity lasted until day 49 in cycle 4 . The very low IgG ADA signal was detected after 7 days after drug administration. (E) For subject 5 a transient ADA IgM course and a weak IgG ADA signal was detected. (F) For subject 6 high IgM ADA and IgG ADA response was detected in cycle 1. The immunoglobulin class switch is demonstrated in cycle 2 on day 28.

AU: Absorbance unit; ADA: Anti-drug antibody; BLQ: Below limit of quantification; CP: Cut point; d: Day; NC: Negative control; TIC: Targeted immunocytokine.

using the IgM and IgG AIA to characterize the immunoglobulin isotype and to demonstrate the class switch of ADAs. During the assay qualification, the assay drug tolerance resulted in $1250 \mathrm{ng} / \mathrm{ml} \mathrm{IgM} \mathrm{ADA} \mathrm{at} 12.5 \mu \mathrm{g} / \mathrm{ml}$ TIC. However, during the case study, the drug levels were below $6.92 \mu \mathrm{g} / \mathrm{ml}$ and the IgM AIA signals was not affected as signals up to $3.0 \mathrm{AU}$ were determined as shown in Figure 3A. Out of 18 subjects with confirmed ADA positive samples, 11 subjects had both, $\operatorname{IgM}$ and $\operatorname{IgG}$ positive responses. For subjects 1, 2 and 6 (Figure 3A, B \& F) as well as for subject 7 (Supplementary Figure 1A) the immunoglobulin class switch is shown. Furthermore, for subjects 1 and 2 (Figure 3A \& B) a persistent IgG ADA up to cycle 20 is determined. 
Interestingly for four subjects solely IgM ADAs were detected. Exemplarily the results of the subject 3 as shown in Figure 3C indicate no IgG AIA signal above the assay cut point over 19 treatment cycles. For the other three subjects, weak IgM AIA signals were detected up to cycle 2.

Weak or borderline IgM and IgG AIA assay signals are depicted in Supplementary Figures 1 \& 2. For eight subjects a weak immune response up to cycle 4 were shown Supplementary Figure 1. For four subjects, the results of IgM and IgG AIA signals were borderline or below detection limit as demonstrated in Supplementary Figure 2. For 10 from 12 subjects with weak ADA IgM response, the assay signal was below 1 AU (Supplementary Figures 1 $\& 2$ ). For these subjects, also the ADA screening assay resulted in low signals and borderline responses with similar profile. Therefore, we conclude that the sensitivity of ADA IgM IgG assays was adequate to detect any specific immune response to TIC.

For the subjects with low immune response as demonstrated in Supplementary Figures $1 \& 2$, it is unclear how the immune response would change in course of time after the treatment cycle 2. However, the comparison of the cycle 2 results in patients with high versus low signal clearly show the different intensity of the individual immune response in subjects. Although for majority of these patients (ten of 12), a low or borderline screening assay signal was observed.

In summary IgM AIA allowed to further investigate the ADA IgM response for 18 individuals in a straight forward manner. For the majority of the subjects, the IgM onset was observed in cycle 1, 4 days after the first drug administration. This was in line with ADA results obtained from the first ADA assessment tier proving that this assay is adequately detecting clinically relevant $\mathrm{ADAs}$ of the IgG and the IgM class. IgM AIA signal intensities were diverse and ranged from strong, moderate and weak to borderline.

\section{Conclusion}

The isotyping of ADA plays a role for assessing and understanding the immune response profile after administration of biopharmaceuticals in preclinical or clinical studies and supports the differentiation between drug treatmentinduced transient and persistent immune responses by separate detection of ADAs of the IgM and the IgG class.

During IgM AIA development, the key assay parameters have been investigated. The evaluation of the qualification experiments shows the assay has acceptable sensitivity, is precise and specific and fulfills the assay acceptance criteria as described in section general method descriptions. Due to the use of LCB and an MRD of 100 the matrix interferences were reduced to acceptable range.

One critical assay reagent for the ADA assay development is the identification and generation of a suitable PC. In this study, two different approaches for generation of surrogate PCs, a conjugated and a recombinant, were evaluated. Both reagents were proven to be suitable for the IgM AIA assay and showed comparable and reproducible results.

The key advantages of the recombinant PC with the developed assay system are that it is a well-defined molecule, the structure is more comparable with endogenous $\operatorname{IgM}$ or $\operatorname{IgG}$ and it is available in large amounts and constant quality. The challenges of this approach are the feasibility of monoclonal anti-idiotypic IgM antibody generation and the more complex purification process.

The key advantages of a conjugated PC are the simple protein chemistry, the feasibility of monoclonal and polyclonal anti-idiotypic antibody generation and the fast production. The challenges of this approach are the higher batch-to-batch variability and the heterogeneous mixture of conjugates with a broad molecule size range resulting in a relatively low product yield.

The manufacturing process of a recombinant PC leads to a well-defined molecule structure with a high batch-tobatch comparability. This is an advantage for the long term supply of reagents for assays in life cycle management of biopharmaceuticals.

In a case study, the qualified IgM AIA with recombinant PC has proven to detect different courses and intensities of early immune responses. In combination with IgG AIA the immunoglobulin class switch was shown. These results prove our first tier clinical immunogenicity assessment. In addition to the primary ADA detection assay package, the combination of the IgM and IgG AIA is a valuable tool for characterization of ADAs during the clinical immunogenicity assessment. The isotyping results bring insights into the primary and secondary immune response and help to understand and demonstrate the individual ADA formation and the immunoglobulin class switch.

IgM AIA delivers an essential component to immunogenicity assessment package and enables to characterize the early ADA occurrence and in combination with IgG AIA to demonstrate immune response class switch. This work 
was conducted to show the feasibility and applicability of IgM and IgG AIA for ADA positive samples of a clinical study. For some biotherapeutics, it is recommended to further investigate the ADA composition not only for IgM but also for IgG1, IgG4 and IgE [4]. Understanding the isotype composition of ADA response may bring holistic insights to efficacy and safety evaluation of the biotherapeutics.

\section{Future perspective}

In early clinical stage, the conduction of the $\operatorname{IgM} / \operatorname{IgG}$ isotyping may help to understand more about immunogenicity of the molecule and progression of the immune response. The results may be used for investigation of the immunogenic capabilities of biopharmaceuticals and help to improve the development processes and design of less immunogenic molecules and formulations.

For the generation of the surrogate ADA PC, as a critical assay tool, the capability of the laboratory needs to be considered. Whereby, we recommend the use of recombinant PC for IgM isotyping. Furthermore, the use of study specific cut point for IgM/IgG isotyping should be considered. Multiplexing techniques for ADA isotyping may remarkably reduce the analysis efforts and the required sample volume during the clinical study phases.

\section{Executive summary}

\section{Background}

- An anti-drug antibody (ADA) characterization assay was developed to detect IgM antibodies in ADA confirmed samples.

- The development of an IgM detection assay is challenging due to the size, structure and low affine unspecific antigen binding properties of IgM antibodies.

Experimental

- For the positive control (PC) as one of the critical assay reagents, a chemically conjugated and a recombinant PC, were tested.

Results \& discussion

- In the course of the assay development, appropriate microtiter plates, assay buffer and capture/detection reagents and their concentrations were evaluated.

- The assay was qualified based on the current guidelines, and the sensitivity, selectivity, specificity, minimum required dilution and drug tolerance were determined.

- In a clinical Phase I study with subjects receiving a human targeted immunocytokine, 18 subjects were confirmed positive for ADAs. These samples were furthermore characterized by using IgM/lgG anti-drug antibody isotyping assay. Individual profiles for IgM and IgG immune response with different signal intensities were demonstrated.

Conclusion

- IgM ADA isotyping assay enabled successful characterization of ADA positive samples obtained from patients enrolled in a clinical study. Recombinant IgM PC was proven as suitable and key assay reagent for the first time.

\footnotetext{
Supplementary data

To view the supplementary data that accompany this paper please visit the journal website at: www.futurescience.com/doi/suppl/10.4155/bio-2020-0308
}

\section{Acknowledgments}

The authors thank all involved colleagues from Large Molecule Bioanalytical R\&D and Pharmaceutical Sciences Division for inspiring discussions and review. $\mathrm{R}$ Vogel from Roche Diagnostics $\mathrm{GmbH}$ and his team for providing labelled assay reagents. The authors thank also $\mathrm{K}$ Schutz and A Buehler from RICB and M Zadak from RICM, for supporting in study sample management and sample analysis. In addition, we would like to thank $\mathrm{N}$ Jelinek for the diligent work on the manuscript.

\section{Financial \& competing interests disclosure}

The authors have no relevant affiliations or financial involvement with any organization or entity with a financial interest in or financial conflict with the subject matter or materials discussed in the manuscript. This includes employment, consultancies, honoraria, stock ownership or options, expert testimony, grants or patents received or pending, or royalties.

Writing assistance was utilized in the production of this manuscript. Roche provided Jelinek Scientific Documentation GmbH, Winterthur, Switzerland (JSD) a study concept paper containing first drafts of body text passages and data tables/diagrams for writing assistance. Based on this information, JSD prepared a first draft of the paper (text, tables and figures) taking into account the Bioanalysis Author Guidelines, harmonisation of spellings, and consistency of content. 


\section{Ethical conduct of research}

The authors state that they have obtained appropriate institutional review board approval or have followed the principles outlined in the Declaration of Helsinki for all human or animal experimental investigations. In addition, for investigations involving human subjects, informed consent has been obtained from the participants involved.

\section{Open access}

This work is licensed under the Attribution-NonCommercial-NoDerivatives 4.0 Unported License. To view a copy of this license, visit http://creativecommons.org/licenses/by-nc-nd/4.0/

\section{References}

1. European Medicines Agency. Guideline on Immunogenicity Assessment of Therapeutic Proteins (2017). https:

//www.ema.europa.eu/en/documents/scientific-guideline/guideline-immunogenicity-assessment-therapeutic-proteins-revision-1_en.pdf

2. Esser C, Radbruch A. Immunoglobulin class switching: molecular and cellular analysis. Annu. Rev. Immunol. 8(1), 717-735 (1990).

3. Jeffrey KA. Introductory Immunology (2nd Edition). Elsevier (2019).

4. Gorovits B. Current considerations for immunoglobulin isotype characterization of antibody response against biotherapeutics. AAPS J. 22(6), 144 (2020).

5. Montjovent MO, Bütikofer L, Faust H, Wood S, Struwe P. Development, validation and application of ADA isotype specific assays. EBF (2012).

6. Leung S, Fiorotti C, Bibik M, Brown D, Gleeson MA, Gorovits B. Multiplexed immunoassay approach to characterize antidrug antibody like specific reactivity. Bioanalysis 11(8), 703-712 (2019).

7. Niu H, Klem T, Yang J, Qiu Y, Pan L. A biotin-drug extraction and acid dissociation (BEAD) procedure to eliminate matrix and drug interferences in a protein complex anti-drug antibody (ADA) isotype specific assay. J. Immunol. Methods 446, 30-36 (2017).

8. US FDA. Immunogenicity Testing of Therapeutic Protein Products - Developing and Validation Assays for Anti-Drug Antibody Detection (2019). https://www.fda.gov/regulatory-information/search-fda-guidance-documents/immunogenicity-testing-therapeuticprotein-products-developing-and-validating-assays-anti-drug

9. Stubenrauch K, Wessels U, Vogel R, Schleypen J. Evaluation of a biosensor immunoassay for simultaneous characterization of isotype and binding region of human anti-tocilizumab antibodies with control by surrogate standards. Anal. Biochem. 390(2), 189-196 (2009).

10. Chin SE, Ferraro F, Groves M, Liang M, Vaughan TJ, Dobson CL. Isolation of high-affinity, neutralizing anti-idiotype antibodies by phage and ribosome display for application in immunogenicity and pharmacokinetic analyses. J. Immunol. Methods 416, 49-58 (2015).

11. Doenecke A, Winnacker EL, Hallek M. Rapid amplification of cDNA ends (RACE) improves the PCR-based isolation of immunoglobulin variable region genes from murine and human lymphoma cells and cell lines. Leukemia 11(10), 1787-1792 (1997).

12. Tornetta M, Fisher D, O’Neil K et al. Isolation of human anti-idiotypic antibodies by phage display for clinical immune response assays. J. Immunol. Methods 328(1-2), 34-44 (2007).

13. Stubenrauch K, Wessels U, Vogel R, Schleypen J. Evaluation of a biosensor immunoassay for simultaneous characterization of isotype and binding region of human anti-tocilizumab antibodies with control by surrogate standards. Anal. Biochem. 390(2), 189-196 (2009).

14. Backliwal G, Hildinger M, Kuettel I, Delegrange F, Hacker DL, Wurm FM. Valproic acid: a viable alternative to sodium butyrate for enhancing protein expression in mammalian cell cultures. Biotechnol. Bioeng. 101(1), 182-189 (2008).

15. Norderhaug L, Olafsen T, Michaelsen TE, Sandlie I. Versatile vectors for transient and stable expression of recombinant antibody molecules in mammalian cells. J. Immunol. Methods 204, 77-87 (1997).

16. Kishimoto S, Nakashimada Y, Yokota R, Hatanaka T, Adachi M, Ito Y. Site-specific chemical conjugation of antibodies by using affinity peptide for the development of therapeutic antibody format. Bioconjugate Chem. 30(3), 698-702 (2019).

17. Staack RF, Stracke J, Stubenrauch K, Vogel R, Schleypen J, Papadimitriou A. Quality requirements for critical assay reagents used in bioanalysis of therapeutic proteins: what bioanalysts should know about their reagents. Bioanalysis 3(5), 523-534 (2011).

18. Stubenrauch K, Künzel C, Vogel R, Tuerck D, Schick E, Heinrich J. Epitope characterization of the ADA response directed against a targeted immunocytokine. J. Pharm. Biomed. Anal. 114, 296-304 (2015).

19. Kiszel P, Fiesel S, Voit S, et al. Transient gene expression using valproic acid in combination with co-transfection of SV40 large $\mathrm{T}$ antigen and human $21^{\mathrm{CIP}} / \mathrm{p} 27^{\mathrm{KIP}}$. Biotechnol. Prog. 35(3), e2786 (2019).

20. Shankar G, Devanarayan V, Amaravadi L et al. Recommendations for the validation of immunoassays used for detection of host antibodies against biotechnology products. J. Pharm. Biomed. Anal. 48(5), 1267-1281 (2008).

21. Wessels U, Schick E, Ritter M, Kowalewsky F, Heinrich J, Stubenrauch K. Novel drug and soluble target tolerant antidrug antibody assay for therapeutic antibodies bearing the P329G mutation. Bioanalysis 9(11), 849-859 (2017). 
